\title{
Eletrorretinograma: considerações a respeitodos limites de normalidade e comparação entre valores normais de dois diferentes laboratórios
}

\author{
Electroretinogram:comparison of normal values between twolaboratories
}

\author{
Flávio Rocha Lima Paranhos ${ }^{1}$ \\ Augusto Paranhos Jr. ${ }^{2}$ \\ Márcio Bittar Nehemy ${ }^{3}$
}

\footnotetext{
${ }^{1}$ Doutor em Oftalmologia pela Faculdade de Medicina da Universidade Federal de Minas Gerais - UFMG, Chefe do Serviço de Retina e Vítreo do Hospital da Visão em Goiânia, GO, Mestrando em Filosofia na Universidade Federal de Goiás.

${ }^{2}$ Mestre e pós-graduando (nível Doutorado) em Oftalmologia pela Escola Paulista de Medicina, Universidade Federal de São Paulo.

${ }^{3}$ Doutor em Oftalmologia, Professor Adjunto e chefe do Serviço de Vítreo do Hospital São Geraldo, Faculdade de Medicina-Universidade Federal de Minas Gerais, Diretor do Instituto da Visão em Belo Horizonte, MG Endereço para correspondência: Rua 38, 300 - Goiânia (GO) CEP 74150-250.

E-mail: flavioparanhos@uol.com.br

Recebido para publicação em 29.03.2001

Aceito para publicação em 17.07.2001

Nota Editorial: Pela análise deste trabalho e por sua anuência sobre a divulgação desta nota, agradecemos aos Drs. Walter Yukihiko Takahashi e Regina Halfeld Ferreira de Mendonça.
}

\begin{tabular}{|l|}
\hline RESUMO \\
\hline Objetivo: Comparar os valores dos limites de normalidade da amplitude e \\
tempo de culminação do eletrorretinograma(ERG) de 2 laboratórios diferen- \\
tes. Métodos: Dados normativos do ERG disponibilizados pelo fabricante \\
de aparelhos de eletrofisiologia com oprogramaUTAS (LKCTechnologies), \\
que incluíam 110 indivíduos normais de 22 a 79 anos de idade, foram \\
comparados com valores normais de nosso laboratório publicados anterior- \\
mente, referentes a 42 indivíduos normais com idades variando de 6 a 72 \\
anos, cujo exame foi realizado em um dos modelos LKC (EPIC 2000). O \\
método de registro do ERG foi o de estimulação simples ("single flash") e \\
seguiu as recomendações da ISCEV (International Society of Clinical \\
Electrophysiology of Vision). Resultados: Todos os parâmetros do ERG, \\
tanto de amplitude quanto de tempo de culminação, com exceção da \\
amplitude dos potenciais oscilatórios (p=0,0779) e do tempo deculminação \\
da onda-b da resposta máxima combinada(p=0,7771), foram significativa- \\
mente(p<0,0001) diferentes. Conclusão: Cadalaboratóriodeeletrofisiologia \\
deve ter seus próprios parâmetros de normalidade, os quais podem ser \\
resultado do intervalo de confiança de 95\% da média de sua amostra de \\
normais, ou os percentis 2,5 e 97,5. No primeiro caso os limites serão \\
bastante estreitos, aumentando a sensibilidade do teste, ocorrendo o \\
inverso no segundo.
\end{tabular}

Descritores: Eletrorretinografia; Valores de referência; Eletrofisiologia/normas; Intervalos de confiança; Estudo comparativo

\section{INTRODUÇÃO}

A determinação dos valores normais do eletrorretinograma (ERG) é de grande importância para que esse exame seja de real utilidade na prática clínica. Se é verdade que há situações em que sua alteração é tão drástica que prescinde de parâmetros acurados de normalidade, como é o caso da retinose pigmentar típica, com um ERG sem resposta registrável na maioria das vezes ${ }^{(1-2)}$, também o é que há diversas outras situações em que tais parâmetros têm um papel crítico. Na doença de Stargardt, por exemplo, podemos observar apenas uma discreta diminuição da amplitude de resposta do componente b-fotópico ${ }^{(3)}$. A determinação objetiva do grau de isquemia das doenças vasculares ${ }^{(4-5)}$, o estudo de determinadas doenças inflamatórias da retina ${ }^{(6-7)}$, também são exemplos em que os limites de normalidade do laboratório são fundamentais para o diagnóstico e o acompanhamento. Merece nota ainda o chamado ERG "supranormal" (ou "hiperanormal"), 
presente em determinadas maculopatias, neuropatias ópticas e estados inflamatórios e/ou tóxicos ${ }^{(8)}$, o que demonstra a necessidade não só de limites inferiores de normalidade, mas também de limites superiores, que permitam a correta identificação de ocorrências desse tipo.

A ISCEV (International Society of Clinical Electrophysiology of Vision) recomenda que, em virtude da presença de muitas variáveis que podem influir nos resultados do ERG, cada laboratório tenha seus parâmetros de normalidade ${ }^{(9)}$. Por outro lado, alguns fabricantes fornecem tabelas com valores normais para seus aparelhos. A LKC, por exemplo, fabricante de uma linha de aparelhos que utilizam o programa UTAS, e a cúpula de Ganzfeld, com rotina padronizada para todos os seus modelos, coloca à disposição de seus usuários dados normativos da amplitude e do tempo implícito (tempo de culminação ou latência de pico) do ERG coletados a partir de uma amostra de 110 indivíduos normais ${ }^{(10)}$. O objetivo do presente estudo é tecer algumas considerações acerca dos parâmetros de normalidade do ERG, assim como comparar os dados normativos fornecidos pelo fabricante com dados próprios de nosso laboratório(11), obtidos em um de seus aparelhos, o EPIC 2000 (LKC Technologies).

\section{MÉTODOS}

Os dados normativos fornecidos pelo fabricante ${ }^{(10)}$ (LKC Technologies, Inc., Gaithersburg, EUA) foram baseados no registro de resposta do ERG de uma amostra de 110 indivíduos normais, com idades variando entre 22 e 79 anos. Os dados normativos de nosso laboratório ${ }^{(11)}$ foram obtidos a partir de uma amostra de 42 indivíduos normais, com idades variando entre 6 e 72 anos. O fabricante não disponibiliza a média e desvio padrão das idades dos indivíduos de sua amostra.

O método de realização do ERG foi detalhadamente descrito previamente. A seguir, uma descrição resumida:

Utilizamos o EPIC 2000 (LKC Technologies, Inc., Gaithersburg, EUA), com o programa UTAS versão 3.10 e cúpula semiautomática de Ganzfeld, com protocolo recomendado pela (ISCEV), rotina standard. O paciente tem suas pupilas dilata- das com tropicamida a $1 \%$ e fenilefrina a $10 \%$. Vinte minutos de adaptação ao escuro precedem o exame. Após a colocação dos eletrodos "terra" na testa e "referência" no lobo da orelha, instila-se colírio anestésico em ambos os olhos (AO) e aplicam-se os eletrodos ativos (ERG jet electrodes), nas córneas do paciente. O exame constitui-se de 5 etapas, sendo as 3 primeiras no escuro e as 2 últimas no claro: 1- Escotópico B. 2Resposta máxima combinada. 3- Potenciais oscilatórios. 4Fotópico B. 5- Flicker. Os filtros de low cut são ajustados para $0,3 \mathrm{~Hz}$ e os de high cut para $500 \mathrm{~Hz}$, com exceção do passo 3 (registro dos potenciais oscilatórios), quando os filtros de low cut são regulados para $75 \mathrm{~Hz}$. Os resultados do ERG são comparados com dados normativos ${ }^{(11)}$ de nosso laboratório, sendo as amplitudes de resposta transformadas em porcentagens dos valores normais.

A análise estatística foi realizada com os programas Excel versão 5,0 (Microsoft Corporation, E.U.A.) e Quick Statistica versão 4,5 (StatSoft, Tulsa, Oklahoma, E.U.A.). Foi realizado o teste de Student para amostras independentes, tendo sido adotado o nível de significância usual de 5\% (p < 0,05).

\section{RESULTADOS}

Todos os parâmetros do ERG, tanto de amplitude quanto de tempo de culminação, com exceção da amplitude dos potenciais oscilatórios $(\mathrm{p}=0,0779)$ e do tempo de culminação da onda-b da resposta máxima combinada $(\mathrm{p}=0,7771)$, foram significativamente $(\mathrm{p}<0,0001)$ diferentes (TABELA).

\section{DISCUSSÃO}

A padronização dos testes eletrofisiológicos tem sido uma constante preocupação da ISCEV. Não somente os parâmetros de estimulação, mas também os valores de normalidade. A última atualização dos padrões do eletrorretinograma ${ }^{(9)}$ recomenda que cada laboratório tenha seus limites de normalidade, que "abarquem" 95\% das respostas de uma amostra de indivíduos normais. Infelizmente, o texto citado é um tanto ambíguo, pois também sugere que valores de normalidade

Tabela - Comparação entre os valores da média ( \pm 1 desvio padrão) de amplitude (em microvolts) e tempo de latência de pico (em milissegundos) de nosso laboratório (GYN) e do laboratório da LKC Technologies. Teste de student bicaudal.

\begin{tabular}{|c|c|c|c|c|c|c|}
\hline & & Amplitude & & & Latênc & ico \\
\hline & GYN & LKC & (p) & GYN & LKC & (p) \\
\hline Escotópico-b & $241 \pm 64$ & $330 \pm 61$ & $(0,0001)^{*}$ & $85 \pm 7$ & $* *$ & - \\
\hline Resposta Combinada & & & & & & \\
\hline Onda-a & $311 \pm 61$ & $387 \pm 70$ & $(0,0001)^{*}$ & $16 \pm 2$ & $21 \pm 1$ & $(0,0001)^{*}$ \\
\hline Onda-b & $515 \pm 99$ & $644 \pm 117$ & $(0,0001)^{\star}$ & $47 \pm 6$ & $48 \pm 3$ & $(0,7771)$ \\
\hline Potenciais Oscilatórios & $254 \pm 75$ & $235 \pm 50$ & $(0,0779)$ & - & - & - \\
\hline Fotópico-b & $212 \pm 69$ & $183 \pm 37$ & $(0,0014)^{*}$ & $31 \pm 2$ & $30 \pm 2$ & $(0,0001)^{*}$ \\
\hline Flicker & $153 \pm 45$ & $133 \pm 30$ & $(0,0021)^{*}$ & $27 \pm 2$ & $27 \pm 2$ & $(0,0001)^{*}$ \\
\hline
\end{tabular}


devam corresponder ao limites de confiança de 95\%. "Limites de confiança" podem ser interpretados como sinônimo de "intervalo de confiança"(12). Ora, "intervalo de confiança de 95\%" não é o mesmo que "abarcar 95\% das respostas normais". Este significa considerar os percentis 2,5 e 97,5 como limites de normalidade, enquanto aquele significa "que se pode estar $95 \%$ confiante de que a verdadeira média encontra-se naquele intervalo". Admitindo-se uma distribuição gaussiana para as amostras, poderíamos dizer que a primeira situação equivaleria a se considerar como limites de normalidade a média $\pm 1,96$ desvios padrão (DP), enquanto a segunda equivaleria à média $\pm 1,96$ erros padrão, o que, absolutamente, não é a mesma coisa. $\mathrm{O}$ desvio é sempre muito maior do que o erro padrão. Em conseqüência, o primeiro critério tornaria os limites de normalidade significativamente mais elásticos (e, portanto menos sensíveis) do que o segundo, criando situações paradoxais, como a que aconteceu com Lemos ${ }^{(6)}$ e Teixeira ${ }^{(7)}$. Esses autores encontraram diferença significativa quando compararam sua amostra de pacientes com uveíte intermediária e Síndrome de Vogt-Koyanagi-Harada, respectivamente, com uma amostra de 57 normais pelo teste de ANOVA Kruskal-Walis. Entretanto, ao distribuir sua amostra pelos percentis, e considerando como limites de normalidade os percentis 5 e 95, a maioria dos pacientes seria considerada como "normal". Costa e col.(13), por sua vez, apresentaram seus resultados do ERG realizado pelo aparelho NICOLET Compact Four em 40 indivíduos normais, considerando tanto o critério da média $\pm 2 \mathrm{DP}$, quanto o intervalo de confiança de 95\% inadequados, preferindo adotar os valores mínimo e máximo, ou seja, um critério ainda mais elástico do que os percentis 2,5 e 97,5. Heckenlively e col. ${ }^{(8)}$ e Matsui e col..$^{(4)}$ publicaram seus valores normais para o ERG considerando a média $\pm 2 \mathrm{DP}$. Heckenlively e col. ${ }^{(8)}$ justificaram sua escolha a partir da observação de que sua amostra possuía uma distribuição semelhante à gaussiana em todos os componentes do exame.

Em nosso laboratório, ainda consideramos como limites de normalidade o intervalo de confiança de $95 \%$ de nossa amostra estudada. O resultado de cada paciente individualmente é comparado com o limite inferior, se menor, ou superior, se maior, e dado em forma de porcentagem do normal, como fazem Matsui e col. ${ }^{(4)}$. Para possibilitar a comparação entre nosso laboratório e os dados normativos fornecidos pelo fabricante, utilizamos a média e o desvio padrão para os cálculos estatísti$\cos$. Tivemos assim a oportunidade de observar que a maioria das diferenças foi significativa, particularmente quanto à amplitude de resposta, cuja variabilidade é sabidamente maior do que o tempo implícito. Apesar de não ter sido possível comparar as médias de idade dos dois laboratórios, os limites de idade das amostras foram semelhantes (6 a 72 anos e 22 a 79 anos), o que nos permite uma comparação dos valores normais de ERG razoavelmente justa. Além disso, já tivemos oportunidade de observar a não correlação entre a amplitude de resposta do ERG e a idade, particularmente antes dos 40 anos $^{(11)}$. Acreditamos que tal achado reforça a recomendação da ISCEV para que cada laboratório tenha seus próprios limites de normalidade. Ao se adquirir um aparelho de eletrofisiologia, devese estar atento a esse detalhe, procurando utilizar dados normativos fornecidos pelo fabricante apenas enquanto ainda não se possui os próprios. Quanto a considerar como normal o "intervalo de confiança de 95\%" ou "os percentis 2,5 e 97,5", acreditamos que o examinador deve lançar mão do bom senso, pois cada critério tem sua vantagem e desvantagem. O primeiro ganha em sensibilidade, mas pode perder em especificidade, acontecendo o contrário com o segundo. É provável que, à medida que se aumente o tamanho da amostra, haja uma diminuição da variação dos resultados, principalmente de amplitude, permitindo que se utilizem os percentis sem grande prejuízo à sensibilidade do teste.

\section{CONCLUSÃO}

Cada laboratório de eletrofisiologia deve ter sua própria padronização dos limites de normalidade do eletrorretinograma, resultado da estatística descritiva de uma amostra representativa da população a que serve o laboratório. Pode-se optar pelo intervalo de confiança de $95 \%$ da média de sua amostra de normais, ou os percentis 2,5 e 97,5. No primeiro caso os limites serão mais estreitos, aumentando a sensibilidade do teste, ocorrendo o inverso no segundo.

\section{ABSTRACT}

Purpose: To compare the limits of normality of the electroretinogram (ERG) amplitude and implicit time of two different laboratories. Methods: Normative ERG data from LKC Technologies, which uses UTAS software were compared to normative data from our laboratory using LKC equipment (EPIC 2000). The former included 110 normal subjects with ages ranging from 22 to 79 years and the latter included 42 normal subjects ranging from 6 to 72 years of age. Single flash ERG using ISCEV (International Society of Clinical Electrophysiology of Vision) recommended routine with ERG-jet contact lens electrodes were performed in both laboratories. Results: All ERG amplitude parameters, as well as all implicit times were significantly different $(\mathrm{p}<0.0001)$ between both laboratories with the exception of the oscillatory potentials' amplitudes $(\mathrm{p}=0.0779)$ and the $\mathrm{b}$-wave implicit times of the maximal combined response $(p=0.7771)$. Conclusions: Each electrophysiology laboratory should have its own normal limit standardization, which can be determined either by the $95 \%$ confidence interval of its normal data sample, or the 2.5 and 97.5 percentiles. The former is narrower and therefore more sensitive, the latter has a much broader range and therefore is significantly less sensitive.

Keywords: Electroretinography; Electrophysiology/standards; Reference values; Confidence intervals; Comparative study 


\section{REFERÊNCIAS}

1. Paranhos FRL, Nehemy MB, Hirose T. Bilateralidade, simetria e correlação clínico-eletrofisiológica na retinose pigmentar. Arq Bras Oftalmol 1998;61:386-9.

2. Paranhos FR, Katsum O, Arai M, Nehemy MB, Hirose T. Pattern reversal visual evoked response in retinitis pigmentosa. Doc Ophthalmol 1999;96:321-31.

3. Itabashi R, Katsumi O, Mehta MC, Wajima R, Tamai M, Hirose T. Stargardt's Disease-fundus flavimaculatus: psychophysical and electrophysiological results. Graefe's Arch Clin Exp Ophthalmol 1993;231:555-62.

4. Matsui Y, Katsumi O, Mehta MC, Hirose T. Correlation of electroretinographic and fluorescein angiographic findings in unilateral central retinal vein obstruction. Graefe's Arch Clin Exp Ophthalmol 1994;232:449-57.

5. Sakaue H, Katsumi O, Hirose T. Electroretinographic findings in fellow eyes of patients with central retinal vein occlusion. Arch Ophthalmol 1989;107:1459-62.

6. Lemos SR. Eletrofisiologia ocular na uveíte intermediária [tese]. Belo Horizonte: Universidade Federal de Minas Gerais, 1999.
7. Teixeira RMB. Eletrofisiologia ocular na síndrome de Vogt-Koyanagi-Harada [tese] Belo Horizonte: Universidade Federal de Minas Gerais, 1999.

8. Heckenlively JR, Tanji T, Logani S. Retrospective study of hyperabnormal (supranormal) electroretinographic responses in 104 patients. Trans Am Ophthalmol Soc 1994;92:218-33

9. Marmor MF, Zrenner E. ISCEV Standard for clinical electroretinography (1999 update). International Society for Clinical Electrophysiology of Vision. Doc Ophthalmol 1999;97:143-56.

10. LKC. The Leader in the electrophysiology of vision. Normative data for the clinical electroretinogram. Gaithersburg MD;1997.

11. Paranhos FRL, Ávila MP, Paranhos A, Cialdini AP. Estudo estatístico de valores normais do eletrorretinograma. Contribuição à padronização do exame. Arq Bras Oftalmol 1997;60:278-84.

12. Elston RC, Johnson WD. Estimates and confidence limits. In: Elston RC, Johnson WD. Essentials of biostatistics. 2 ${ }^{\text {nd }}$. Ed. Philadelphia: F.A. Davis.; 1994. p.113-34.

13. Costa RHM, Takahashi WY, Dias NT. Normatização do eletrorretinograma. Rev Bras Oftalmol 1998;57:375-8.

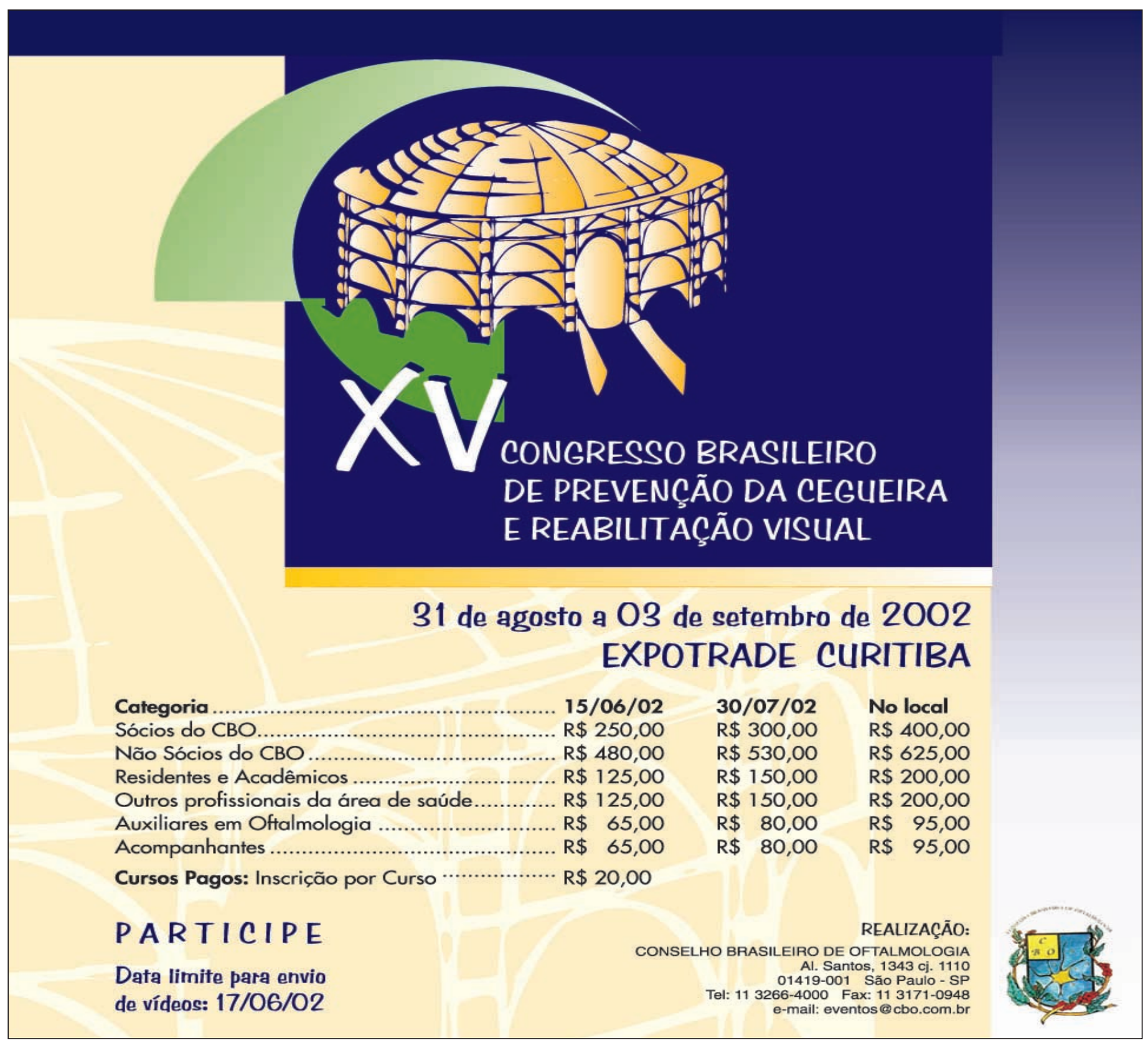

